\title{
Review of: "Glycyrrhizic acid ameliorates submandibular gland oxidative stress, autophagy and vascular dysfunction in rat model of type 1 diabetes"
}

\section{ERSIN DEMIR}

Potential competing interests: The author(s) declared that no potential competing interests exist.

Report: Glycyrrhizic acid ameliorates submandibular gland oxidative stress, autophagy and vascular dysfunction in rat model of type 1 diabetes

This work (Ventimiglia et al.) is not on the reference list.

Information on the preparation of glycyrrhizic acid should be included in the study.

Information about the purity of glycyrrhizic acid should be included.

How did you determine the dose of application of glycyrrhizicn acid? 